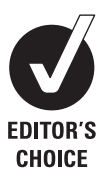

'Department of Rheumatology, Rheumatology Research and Advanced Therapeutics, Radboud University Nijmegen Medical Centre, Nijmegen, The Netherlands

2EA4222, Physiology and Biotherapies of Rheumatoid Arthritis, University of Paris, Paris, Bobigny, France

\section{Correspondence to}

$\mathrm{FA} J$ van de Loo, Rheumatology

Research and Advanced

Therapeutics, Radboud

University Nijmegen

Medical Centre, Nijmegen

Centre for Molecular Life

Sciences, Geert Grooteplein

26-28, 6500 HB Nijmegen,

The Netherlands;

a.vandeLoo@reuma.umcn.n

Accepted 12 July 2011

Published Online First

27 August 2011

\title{
A pivotal role for antigen-presenting cells overexpressing SOCS3 in controlling invariant NKT cell responses during collagen-induced arthritis
}

\author{
Sharon Veenbergen, ${ }^{1}$ Miranda B Bennink, ${ }^{1}$ Alsya J Affandi, ${ }^{1}$ Natacha Bessis, ${ }^{2}$ \\ Jérôme Biton, ${ }^{2}$ Onno J Arntz, ${ }^{1}$ Wim B van den Berg, ${ }^{1}$ Fons A J van de Loo ${ }^{1}$
}

\begin{abstract}
Objective Suppressor of cytokine signalling (SOCS) proteins constitute a class of intracellular proteins that are key physiological regulators of immune cell function. It has previously been shown that antigen-presenting cells (APCs) overexpressing SOCS3 steer T helper immune responses and protect against experimental arthritis. A study was undertaken to investigate the contribution of SOCS3 in regulating invariant natural killer T (iNKT) cell responses during collagen-induced arthritis (CIA).

Methods DBA/1 mice were immunised with type II collagen and adenoviruses encoding SOCS3 were administered intravenously before the clinical onset of arthritis. Murine APCs overexpressing SOCS3 were co-cultured with an iNKT cell hybridoma and interleukin 2 (IL-2) release was measured by Luminex multi-analyte technology. The frequency and activation of primary iNKT cells was assessed by flow cytometry. Murine APCs were analysed for cytokine and CD1d expression following viral SOCS3 gene transfer.
\end{abstract}

Results Viral overexpression of SOCS3 in APCs resulted in reduced activation of the iNKT cell hybridoma. Importantly, during initiation of CIA, adenovirus-mediated overexpression of SOCS3 in hepatic and splenic APCs inhibited iNKT cell expansion in both organs. The iNKT cell population from SOCS3-treated mice showed low expression of the early activation marker CD69 and primary liver iNKT cells produced less interferon $\gamma$ and IL-4 upon $\alpha$-galactosylceramide stimulation. No differences in CD1d surface expression were observed, but SOCS3-transduced APCs produced decreased levels of proinflammatory cytokines and increased levels of IL-10. Conclusion These results demonstrate a critical role for SOCS3 in controlling the immunostimulatory capacities of APCs, which has direct implications for the effector function of iNKT cells during arthritis.

\section{INTRODUCTION}

Invariant natural killer $\mathrm{T}$ (iNKT) cells are a unique subset of thymus-derived $\mathrm{T}$ lymphocytes defined by their co-expression of NK receptors (eg, NK1.1) along with a T cell antigen receptor. ${ }^{1}$ This innate-like lymphocyte population in mice expresses a conserved semi-invariant $\mathrm{T}$ cell receptor (TCR) which is comprised of a V $\alpha 14-J \alpha 18$ TCR chain associated with a V $\beta 8.2$, V $\beta 7$ or, to a lesser extent, V $\beta 2$ chain. ${ }^{2}$ The biased TCR repertoire gives iNKT cells the ability to recognise glycolipid antigens presented by the major histocompatibility complex class I-like molecule CD1d. ${ }^{4}$ The marine sponge-derived glycosphingolipid $\alpha$-galactosylceramide ( $\alpha$-GalCer) was the first synthetic ligand identified as a potent stimulatory agent for iNKT cells. ${ }^{5}$ Resting iNKT cells have a memory or partially activated phenotype reflected by the expression of several memory/ activation markers (CD69, CD44) and the rapid production of an array of cytokines including interferon $\gamma(\mathrm{IFN} \gamma)$, interleukin 2 (IL-2), IL-4, IL-10 and IL-17 upon TCR engagement. ${ }^{6}$ Once activated, iNKT cells can modulate the innate immune response via dendritic cells (DCs), macrophages and NK cells, and have a critical role in the regulation of subsequent adaptive $\mathrm{T}$ and $\mathrm{B}$ cell immune responses. 7

Because of the functional discrepancy of producing both proinflammatory and anti-inflammatory cytokines, iNKT cells can exert both effector and regulatory functions. Evidence has been provided that iNKT cells play a critical role in autoimmune arthritis, either by enhancing disease or by protecting against disease. ${ }^{8}$ Animal studies have shown that prophylactic and semi-therapeutic treatment with $\alpha$-GalCer attenuates the clinical and histopathological severity of collagen-induced arthritis (CIA), a well-established animal model of human rheumatoid arthritis. ${ }^{10}$ By contrast, progression of CIA appears to be less pronounced in J $\alpha 18^{-/-}$ and $\mathrm{CD} 1 \mathrm{~d}^{-/-}$mice or after anti-CD1 treatment. ${ }^{11}$ Moreover, antibody-induced arthritis in the $\mathrm{K} / \mathrm{BxN}$ serum transfer model is found to be less severe in $\mathrm{CD}_{1 \mathrm{~d}^{-/-}}$mice, whereas stimulation of iNKT cells by in vivo administration of $\alpha$-GalCer promotes joint swelling. ${ }^{12}$ Considering the dual functionality of iNKT cells in arthritis development and severity, it is tempting to exploit immunoregulatory mechanisms to steer the iNKT cell immune response during the disease process. Nowadays, structural analogues of $\alpha$-GalCer have received considerable interest for their potential to control iNKT cell immune responses. ${ }^{13}$

Members of the suppressor of cytokine signalling (SOCS) family are pivotal negative intracellular regulators of cytokine and Toll-like receptor (TLR) responses, including those that regulate innate and adaptive immune responses. Evidence accumulated over the last decade strongly points to an important role for SOCS3 in the regulation of immune cell development and function. ${ }^{14-16}$ We recently demonstrated that intravenouslyinduced overexpression of SOCS3 is effective in preventing CIA. The underlying mechanism involves the generation of tolerogenic antigenpresenting cells (APCs), which eventually affects the outcome of adaptive $T$ helper cell responses. ${ }^{17}$ 
Considering the important immunoregulatory properties of iNKT cells and their dual role in arthritis, we hypothesised that a biased iNKT cell population might be involved in the prophylactic effect of SOCS3 on arthritis. In this study we explored in more detail the ability of APCs overexpressing SOCS3 to regulate iNKT cell responses in vitro and after type II collagen challenge in vivo.

\section{MATERIALS AND METHODS} Mice

Male 10-12-week-old DBA/1 mice (Elevage Janvier, Le Genest Saint Isle, France) were housed in top-filter cages and fed a standard diet with freely available water and food. Mice injected with adenoviral vectors were housed in low-pressure isolator cages. All in vivo studies complied with national legislation and were approved by the local authorities for the care and use of animals with related codes of practice.

\section{Cell lines}

The iNKT hybridoma DN32.D3 cells are derived from C57BL/6 NK1.1+ thymocytes, express the Vo14J $\alpha 18$ rearrangement and show strong reactivity towards CD1d glycolipid antigens. ${ }^{18}$ Cell lines were cultured in Dulbecco's modified Eagle's medium (Invitrogen, Breda, The Netherlands) supplemented with 2\% heat-inactivated fetal calf serum, penicillin-streptomycin (Lonza, Basel, Switzerland), $1 \times$ non-essential amino acids (Invitrogen) and $1 \mathrm{mM}$ pyruvate.

\section{Construction of viral vectors}

Third-generation self-inactivating lentiviral vectors containing the human phosphoglycerate kinase (PGK) promoter were used for generation of recombinant lentiviral vectors according to the method described previously. ${ }^{19}$ Adenoviral vectors were E1A, B and E3 deleted and produced according to the AdEasy system method as described previously. ${ }^{20} 21$

\section{In vitro stimulation assays}

One day before transduction, J774 macrophages were seeded in a 96 -well plate $\left(1.5 \times 10^{5} /\right.$ well). Cells were transduced with $250 \mathrm{ng}$ p24gag equivalents lentivirus in medium supplemented with $8 \mu \mathrm{g} / \mathrm{ml}$ polybrene (Sigma, Zwijndrecht, The Netherlands) for $4 \mathrm{~h}$ at $37^{\circ} \mathrm{C}$. Forty-eight hours after transduction the cells were washed extensively with sterile phosphate-buffered saline (PBS) after which the iNKT cell hybridoma DN32.D3 (5×104/well), lipopolysaccharide (LPS) and/or synthetic $\alpha$-galactosylceramide (KRN7000; Biomol International, Antwerp, Belgium) were added for the next 24 h. For blocking experiments, J774 macrophages were preincubated with $10 \mu \mathrm{g} / \mathrm{ml}$ anti-CD1d blocking antibody or appropriate isotype control (Biolegend, San Diego, California, USA) for $0.5 \mathrm{~h}$ at $4^{\circ} \mathrm{C}$ before the iNKT cell hybridoma and stimulants were added.

\section{Induction of collagen-induced arthritis}

Mice were immunised intradermally at the tail base with $100 \mu \mathrm{g}$ bovine collagen type II (BCII) emulsified in complete Freund's adjuvant (CFA) (Mycobacterium tuberculosis strain H37Ra; Difco Laboratories, Detroit, Michigan, USA) as described previously. ${ }^{17}$ At day 21, mice were given an intraperitoneal booster injection of $100 \mu \mathrm{g}$ BCII. One day after the booster injection, immunised mice were given an intravenous injection of $3 \times 10^{8}$ focus-forming units (FFU) adenovirus. Clinical signs of arthritis in paws and ankle joints were macroscopically scored in a blinded manner by two independent observers using a scale ranging from 0 to 2 for each limb, as described previously. ${ }^{17}$

\section{Isolation of splenocytes and liver mononuclear cells}

The adherent cell fraction mainly consisting of macrophages was isolated from the spleen as described previously. ${ }^{17}$ For the stimulation assay, $1.5 \times 10^{5}$ splenic APCs were stimulated for $24 \mathrm{~h}$ with $1 \mu \mathrm{g} / \mathrm{ml}$ LPS. In addition, splenic APCs $\left(1.5 \times 10^{5} /\right.$ well $)$ were co-cultured for $48 \mathrm{~h}$ with the iNKT cell hybridoma DN32. D3 $\left(5 \times 10^{4} /\right.$ well $)$ in the presence of $1 \mu \mathrm{g} / \mathrm{ml}$ LPS and/or $100 \mathrm{ng} /$ $\mathrm{ml} \alpha$-GalCer. The livers were perfused with sodium chloride, mashed and filtered. The cells were resuspended in a 35\% Percoll solution and centrifuged for $25 \mathrm{~min}$ at $750 \times \mathrm{g}$. Liver mononuclear cells (LMNCs) were collected from pellet and red blood cells were removed by osmotic shock. The LMNCs $\left(1.5 \times 10^{5} /\right.$ well $)$ were stimulated with $100 \mathrm{ng} / \mathrm{ml} \alpha$-GalCer for $48 \mathrm{~h}$.

\section{Cytokine measurement in supernatant}

Levels of murine IL-2, IL-4, IL-6, IL-10, IL-12p70, tumour necrosis factor $\alpha$ (TNF $\alpha)$ and IFN $\gamma$ were determined in cell-free culture supernatants using the Luminex multi-analyte technology. The sensitivity of the assay was approximately $1 \mathrm{pg} / \mathrm{ml}$.

\section{Flow cytometric analysis}

Splenic APCs were stained with phycoerythrin (PE)-labelled antimouse CD1d (1:200; eBioscience, San Diego, California, USA). For the analysis of iNKT cells, Fc $\gamma$ receptors were blocked by preincubation with saturating amounts of anti-Fc $\gamma$ RII/III monoclonal antibody (mAb) (BD Pharmingen). Subsequently, cells were stained with APC-labelled CD1d tetramers (1:100) loaded or not with an $\alpha$-GalCer analogue (PBS57; NIH Tetramer Core Facility, Atlanta, Georgia, USA). The cells were then incubated with FITC-labelled anti-mouse TCR $\beta$ (1:200), PE-labelled anti-mouse CD69 (1:100) or appropriate isotype controls (Biolegend). For intracellular cytokine staining, LMNCs were stimulated for $2 \mathrm{~h}$ with $10 \mathrm{ng} / \mathrm{ml}$ phorbol myristate acetate (PMA) and $1000 \mathrm{ng} / \mathrm{ml}$ ionomycin in the presence of GolgiPlug (BD Pharmingen). The cells were stained for extracellular markers, fixed, permeabilised with Cytofix/Cytoperm solution (BD Pharmingen) and subsequently labelled with anti-mouse IFN $\gamma$-PE (1:200), anti-mouse IL-4-PE (1:200) or appropriate isotype controls (Biolegend) in PBS containing $1 \%$ bovine serum albumin, $2 \%$ fetal calf serum, $0.1 \%$ saponin. Stained cells were analysed using FACScalibur (Becton Dickinson, Mountain View, CA, USA) and FlowJo software.

\section{RNA isolation and quantitative PCR analysis}

Total RNA was extracted using TRIzol reagent and reverse transcribed into cDNA as described previously. ${ }^{22} 23$ Quantitative real-time PCR was performed using the ABI/ Prism 7000 sequence detection system (Applied Biosystems, Foster City, California, USA). The PCR protocol was performed as described before. ${ }^{15}$ Quantification of PCR signals was achieved by calculating the difference between the cycle threshold value $(\mathrm{Ct})$ of the gene of interest with the $\mathrm{Ct}$ value of the reference gene GAPDH $(\triangle \mathrm{Ct})$. Primer sets used were GAPDH: Fw: 5'-GGCAAATTCAACGGCACA-3', Rv: 5'-GTTAGTGGGGTCTCGCTCCTG-3'; SOCS3: Fw: 5'-CTGG TACTGAGCCGACCTCTC-3', Rv: 5'-CCGTTGACAGTCTTC CGACAA-3'; TNF $\alpha$ : Fw: 5'-CAGACCCTCACACTCAGATCAT CT-3', Rv:5'-CCTCCACTTGGTGGTTTGCTA-3'; IL-6:Fw: 5'-G GAGCCCACCAAGAACGATAG-3', Rv: 5'-TGGATGGAAGTC TCTTGCAGAGA-3'; IL-10: Fw: 5'-ATTTGAATTCCCTGGGT 
GAGAA-3', Rv: 5'-ACACCTTGGTCTTGGAGCTTATTAA-3'; IL-12p35: Fw: 5'-TGGTGAAGACGGCCAGAGA-3', Rv: 5'-CA GTGGTAAACAGGTCTTCAATGTG-3'; IL-12p40: Fw: 5'-AG CTAACCATCTCCTGGTTTGC-3',Rv:5'-CCACCTCTACAAC ATAAACGTCTTTC-3'; IL-18: Fw: 5'-GCCTCAAACCTTCCA AATCACT-3', Rv: 5'-TTTCCTTGAAGTTGACGCAAGAG-3'.

\section{Statistical analysis}

Significance of differences was determined using the MannWhitney U test performed on GraphPad Prism 4.0 software (GraphPad Software, San Diego, California, USA); p values $\leq 0.05$ were regarded as significant.

\section{RESULTS}

\section{Diminished antigen-dependent activation of iNKT cells by macrophages overexpressing SOCS3}

In order to evaluate whether enhanced SOCS3 expression in APCs could influence iNKT cell responses, we developed an in vitro system for co-culturing murine $\mathrm{J} 774$ macrophages with the iNKT cell hybridoma DN32.D3. The J774 macrophages were transduced with a lentiviral vector encoding the SOCS3 gene. An increase in the expression of SOCS3 mRNA was found $48 \mathrm{~h}$ after transduction (figure 1A) and the use of a GFP-lentiviral vector revealed a transduction efficiency of about $84 \%$ (figure 1B). We next determined the ability of SOCS3-transduced J774 macrophages to activate the iNKT cell hybridoma in response to $\alpha$-GalCer. Interestingly, the $\alpha$-GalCer-reactive iNKT cell hybridoma primed by SOCS3-transduced macrophages produced significantly less IL-2 than those primed by macrophages transduced with control virus (figure 1C).

\section{Macrophages overexpressing SOCS3 inhibit cytokine-driven iNKT cell activation}

In addition to the antigenic stimulation of iNKT cells via CD1dpresented antigens, tissue-specific microenvironments (eg, the local cytokine milieu) can drive the antigen-independent activation of CD1d-restricted $\mathrm{T}$ cells or enhance the weak responses to self-glycolipids. ${ }^{24-26}$ We determined the ability of SOCS3-transduced J774 macrophages to activate the iNKT cell hybridoma in response to the TLR4 agonist LPS. After TLR4 stimulation, control macrophages elicited a dose-dependent increase in IL-2 production by the iNKT cell hybridoma whereas SOCS3-transduced macrophages were significantly less efficient in activating the iNKT cell hybridoma (figure $2 \mathrm{~A}$ ). To exclude the possibility that recognition of self-glycolipids presented by CD1d plays a role in the activation process, blocking $\mathrm{CD} 1 \mathrm{~d} \mathrm{mAb}$ or isotype matched control $\mathrm{mAb}$ was added to the J774-iNKT cell hybridoma co-cultures. Figure 2B shows that blocking of CD1d did not lead to differences in the LPS-induced IL- 2 production. As a control, the potent antigen $\alpha$-GalCer was added to the co-cultures and CD1d mAb completely inhibited
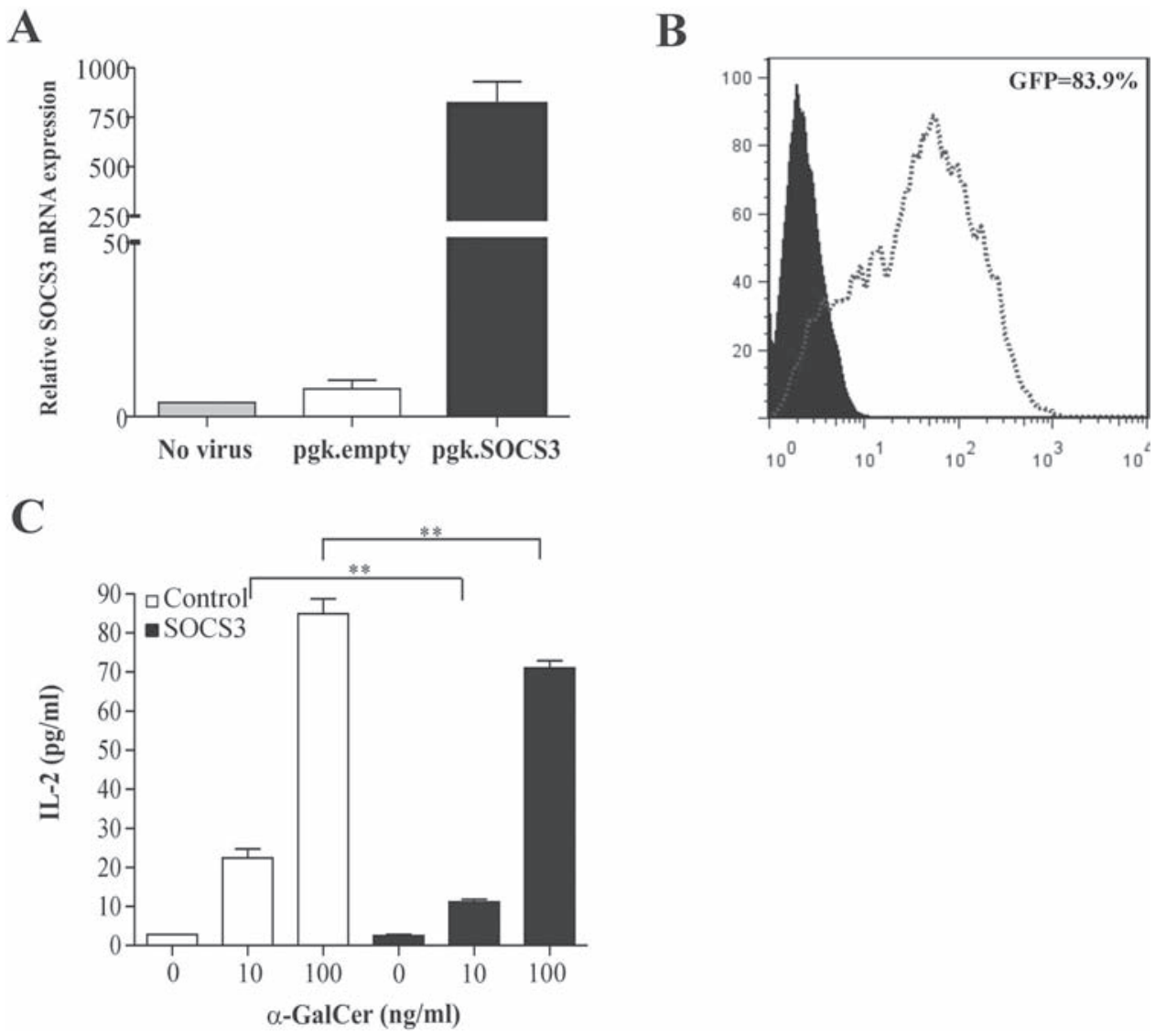

Figure 1 SOCS3-transduced macrophages inhibit the antigen-dependent activation of invariant natural killer T (iNKT) hybridoma cells in vitro. Murine $\mathrm{J} 774$ macrophages were transduced with $250 \mathrm{ng}$ SOCS3-lentiviral vector, an empty lentiviral vector or GFP-lentiviral vector for $4 \mathrm{~h}$ at 37 ${ }^{\circ} \mathrm{C}$. Forty-eight hours after transduction the cells were harvested and (A) SOCS3 mRNA expression was determined by quantitative PCR and (B) green fluorescent protein (GFP) expression was measured by flow cytometry. (C) Murine $\mathbf{J 7 7 4}$ macrophages transduced with an empty lentiviral or SOCS3-lentiviral vector were co-cultured with the iNKT cell hybridoma in the presence of different doses of $\alpha$-GalCer. The levels of IL-2 in cell-free supernatants harvested after $24 \mathrm{~h}$ of co-culture were determined by Luminex multi-analyte technology. Values are mean and SD. The experiments were performed twice with similar results. ${ }^{* *} p<0.01$ vs control. 


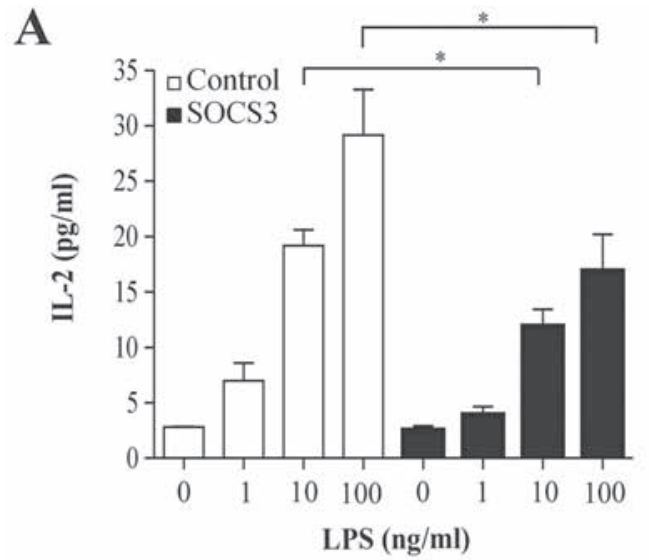

B

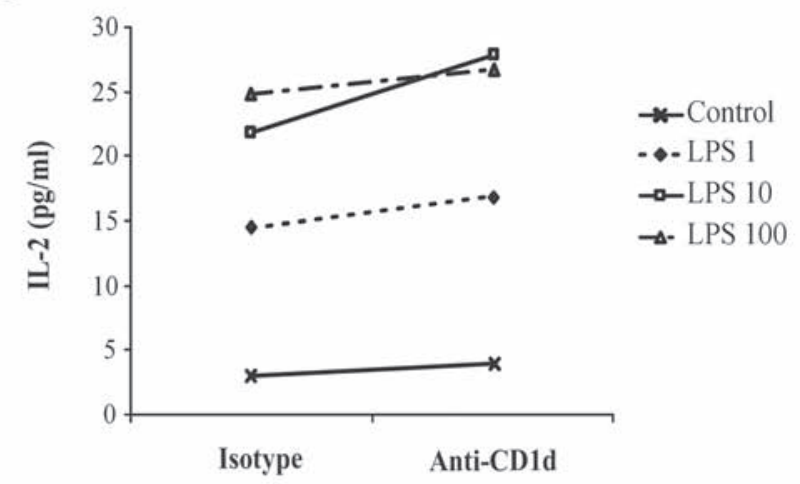

C

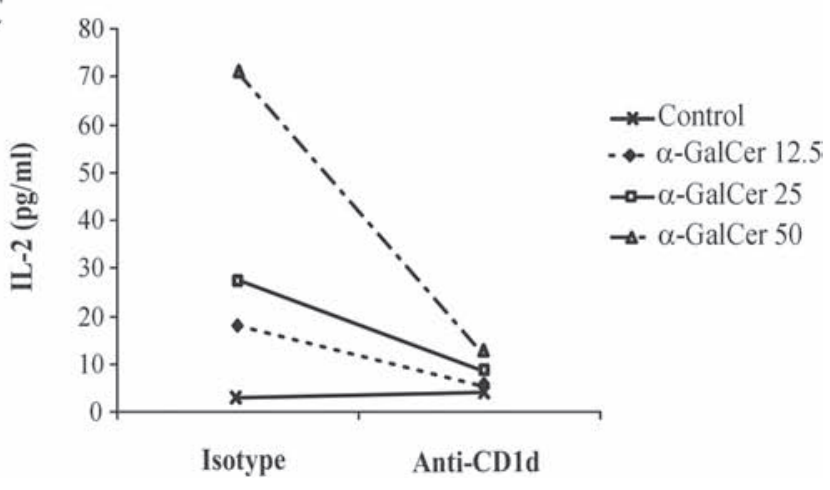

Figure 2 Macrophages overexpressing SOCS3 inhibit the antigenindependent activation of invariant natural killer T (iNKT) hybridoma cells in vitro. (A) Murine J774 macrophages were transduced with 250 ng SOCS3-lentiviral vector or an empty lentiviral vector for $4 \mathrm{~h}$ at $37^{\circ} \mathrm{C}$. Forty-eight hours after transduction the macrophages were co-cultured with the iNKT cell hybridoma in the presence of lipopolysaccharide (LPS). The levels of interleukin 2 (IL-2) in cell-free supernatants harvested after $24 \mathrm{~h}$ of co-culture were determined by Luminex multianalyte technology. Values are mean and SD. The experiments were performed twice with similar results. ${ }^{*} p<0.05$ vs control. (B, C) Murine $\mathrm{J} 774$ macrophages were treated with monoclonal antibody to CD1d or control IgG2 $b$ for $0.5 \mathrm{~h}$ at $4^{\circ} \mathrm{C}$ before adding the iNKT cell hybridoma and different doses of (B) LPS or (C) $\alpha$-galactosylceramide ( $\alpha$-GalCer). The LPS concentrations used were $1,10,100 \mathrm{ng} / \mathrm{ml}$ and the $\alpha$-GalCer concentrations used were $12.5,25,50 \mathrm{ng} / \mathrm{ml}$. The levels of IL-2 in cellfree supernatants were determined after $24 \mathrm{~h}$ of co-culture by Luminex multi-analyte technology.

the antigen-dependent induction of IL-2 production (figure 2C). These results indicate that the iNKT cell-macrophage communication in the LPS-stimulated culture system is not influenced by recognition of self-glycolipids presented by CD1d.
Intravenous delivery of adenovirus-mediated SOCS3 affects the ability of splenic APCs from type II collagen-immunised mice to activate iNKT cells in vitro

Consistent with our previous findings, ${ }^{17}$ intravenous adenoviral transfer of SOCS3 clearly prevented the development of CIA. A clear decreased incidence of arthritis was found during the 5-12day period after virus injection. At day 12 after virus injection, only $40 \%$ of the paws in Ad5.SOCS3-treated mice developed signs of arthritis whereas, in control virus-treated mice, $90 \%$ of the paws were affected (figure $3 \mathrm{~A}$ ). The macroscopic scores of Ad5.SOCS3-treated mice that did become arthritic were the same as those of control virus-treated mice, suggesting that disease severity is identical once the threshold of disease induction has been passed (figure $3 \mathrm{~B}$ ). In this context, the effects of SOCS3 on immune cell function were examined during the 1-3day period after virus injection, before the differences in arthritis incidence occurred. Besides liver resident APCs, we have shown that intravenous injection of adenoviruses leads to enhanced transgene expression in the APC fraction of the spleen, whereas no expression can be found in purified CD3+ T cells. ${ }^{17} 2728 \mathrm{We}$ isolated splenic APCs one day after virus injection and co-cultured them with the iNKT cell hybridoma in the presence of LPS, $\alpha$-GalCer or a combination of both. IL- 2 production was notably increased in the co-cultures containing splenic APCs from control mice following $\alpha$-GalCer or LPS stimulation. Furthermore, the increase in IL-2 secretion by the iNKT cell hybridoma was more pronounced (125-fold) when a combination of $\alpha$-GalCer and LPS was added. The presence of splenic SOCS3-transduced APCs inhibited the increase in LPS, $\alpha$-GalCer and $\alpha$-GalCer plus LPS-induced IL-2 production by $79 \%, 40 \%$ and $55 \%$, respectively (figure 4).

\section{Splenic APCs display an altered cytokine profile but show no differences in CD1d cell surface expression after SOCS3 treatment}

Regulation of CD1d cell surface expression on the APC is one of the mechanisms of controlling antigen-dependent iNKT cell activation and function. ${ }^{29}$ However, no differences in CD1d expression on splenic APCs were found between the control group and the Ad5.SOCS3-treated group 1 and 3 days after virus injection (figure 5A). While CD1d expression was not affected, the splenic SOCS3-transduced APCs produced decreased mRNA and protein levels of TNF $\alpha$, IL- 6 and IL-12, and increased levels of antiinflammatory IL-10 following TLR4 stimulation (figure 5B,C). The TLR4-induced IL-18 mRNA expression was also clearly decreased $(-56 \%)$. The protein levels of IL-18 were below the detection level and no difference in IL-12p40 mRNA expression was found (data not shown). Since the effect of SOCS3 on the antigen-specific activation of iNKT cells cannot be explained by differences in CD1d expression, these results suggest that SOCS3 inhibits both the antigen-dependent and -independent activation of iNKT cells in a cytokine-dependent manner.

\section{SOCS3 treatment controls iNKT cell responses in vivo}

We next investigated the effect of SOCS3 treatment on the primary iNKT cell population after type II collagen challenge in vivo. The percentages and absolute numbers of CD1d tetramerpositive iNKT cells were increased after collagen immunisation in the control virus-treated mice compared with non-immunised mice. Interestingly, intravenous delivery of adenovirus-mediated SOCS3 prevented iNKT cell expansion in both liver and spleen 3 days after virus injection (figure 6A,B). Because our data suggest that SOCS3-transduced APCs inhibit iNKT cell activity, 
A

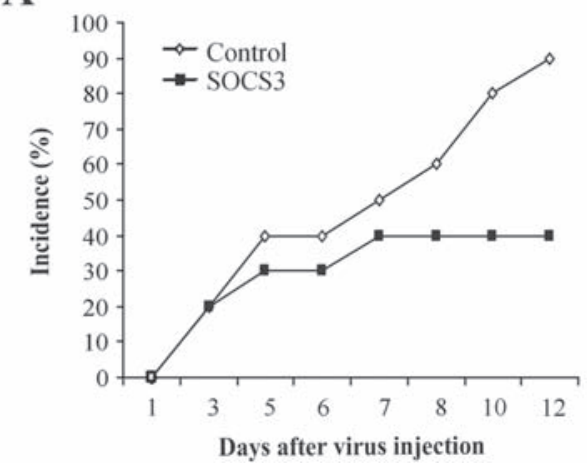

B

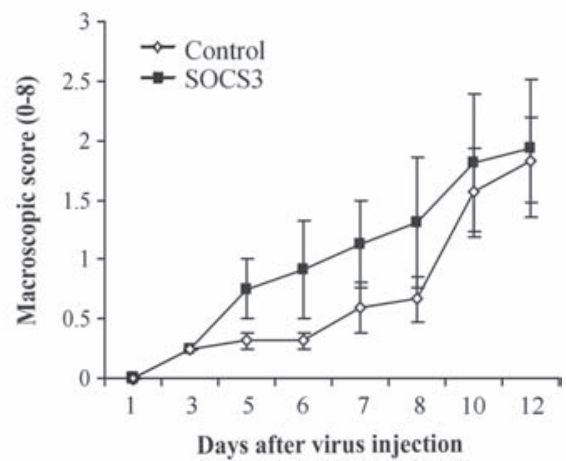

Figure 3 Reduced incidence of arthritis following systemic adenovirus-mediated SOCS3 gene delivery. DBA/1 mice immunised with type II collagen received an intravenous injection of Ad5.SOCS3 or Ad5.luciferase 1 day after the booster injection and before the onset of arthritis. Paws were scored for the development of arthritis by two independent observers. (A) Incidence of arthritis and (B) clinical scores of affected mice are shown. Values are mean and SEM. Representative results of four independent experiments are shown ( $n=10$ mice per group)

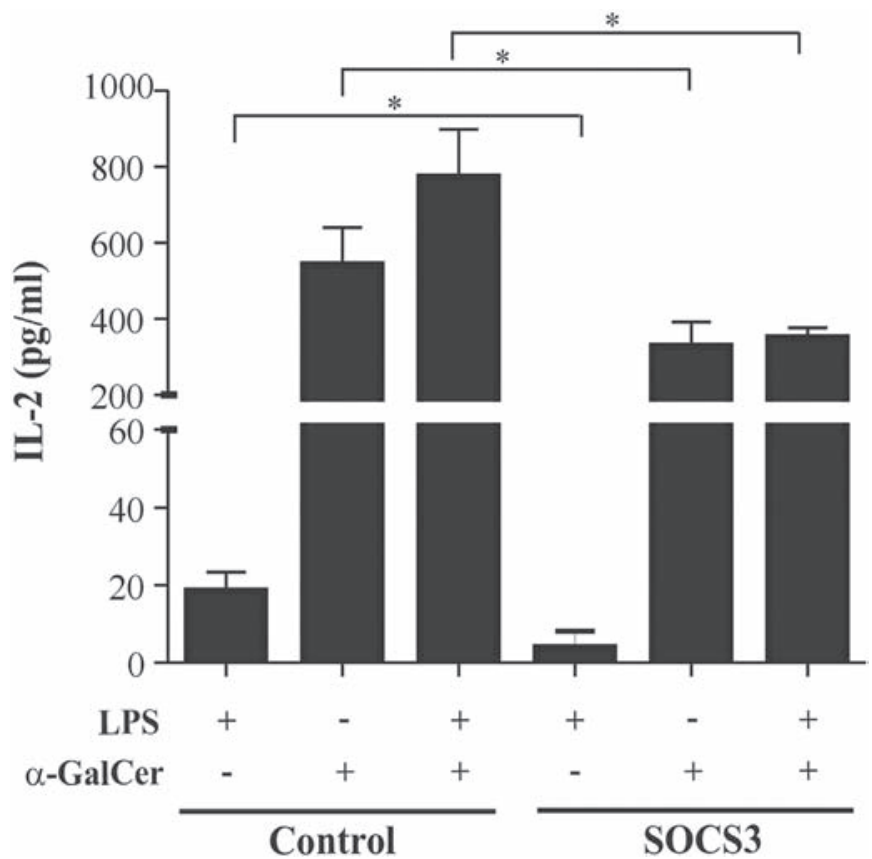

Figure 4 Reduced ability of splenic SOCS3-transduced antigen-presenting cells (APCs) from type II collagen-immunised mice to activate invariant natural killer T (iNKT) hybridoma cells in vitro. Bovine type II collagen-immunised mice were intravenously injected with $3 \times 10^{8}$ focus-forming units (FFU) Ad5.SOCS3 or Ad5.luciferase and killed 1 day later. Primary splenic APCs were cultured with the iNKT cell hybridoma for $48 \mathrm{~h}$ in the presence of $1 \mu \mathrm{g} / \mathrm{ml}$ lipopolysaccharide (LPS) and/or $100 \mathrm{ng} / \mathrm{ml} \alpha$-galactosylceramide ( $\alpha$-GalCer). The levels of interleukin 2 (IL-2) in cell-free supernatants were determined by Luminex multi-analyte technology. Values are corrected for unstimulated controls and depicted as mean plus SEM results from triplicate cultures ( $n=4$ mice). ${ }^{*} p<0.05$ vs control.

we determined the expression of the early activation marker CD69 on the iNKT cell population. A CD69high iNKT and CD69low iNKT cell population was detectable after SOCS3 treatment in both organs, whereas control mice only mainly CD69high iNKT cells. Interestingly, the CD69low iNKT cell population from SOCS3-treated mice expressed CD69 to a similar extent as iNKT cells from non-immunised mice (figure 6C). Frequency analysis clearly showed an increase in splenic iNKT cell percentages during the 1-7-day period after virus injection, and eventually a decrease to the basal level in control virus-treated mice. In contrast, a moderate increase in iNKT cell frequency was found in SOCS3-treated mice (figure 6D). At the same time, in vivo SOCS3 delivery led to reduced numbers of hepatic TCR $\beta+$ cells and iNKT cells that expressed the disease-promoting factor IFN $\gamma$ (figure $6 \mathrm{E}$ ), and LMNCs produced significantly less IFN $\gamma(-67 \%)$ and IL-4 $(-69 \%$ ) upon stimulation with $\alpha$-GalCer (figure $6 \mathrm{~F}$ ). Hepatic
IL-17-positive iNKT cells were not detectable in either control or SOCS3-treated mice, and IL-17 levels were below the detection level in $\alpha$-GalCer-stimulated cultures. Taken together, our findings clearly show that SOCS3 represses iNKT cell responses during the early initiation phase of CIA.

\section{DISCUSSION}

The results of this study identify SOCS3 as an important negative regulator of iNKT cell immune responses. We have shown that APCs genetically modified to overexpress SOCS3 inhibit the antigen-specific and non-specific activation of an iNKT cell hybridoma. In type II collagen-immunised mice, enhanced SOCS3 expression in hepatic and splenic APCs resulted in reduced iNKT cell expansion, low expression of the early activation marker CD69 and reduced production of IFN $\gamma$ and IL-4 by LMNCs upon $\alpha$-GalCer stimulation. The mechanism underlying 
A

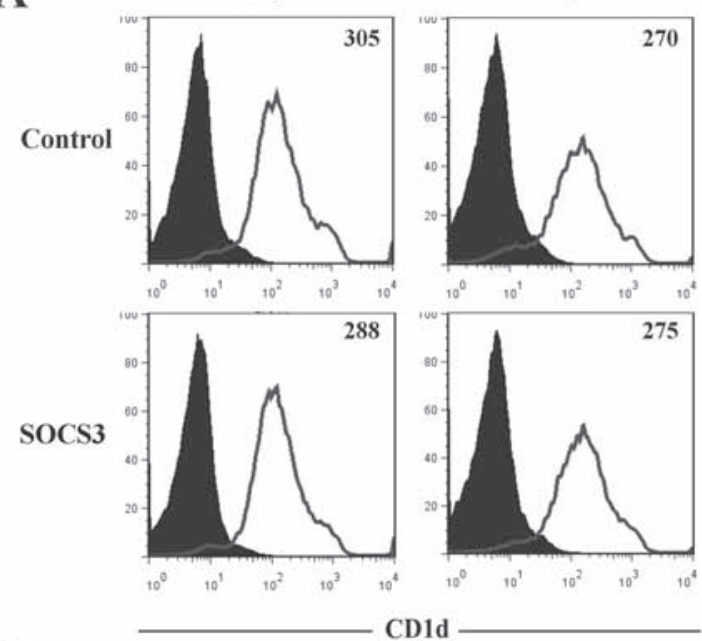

B
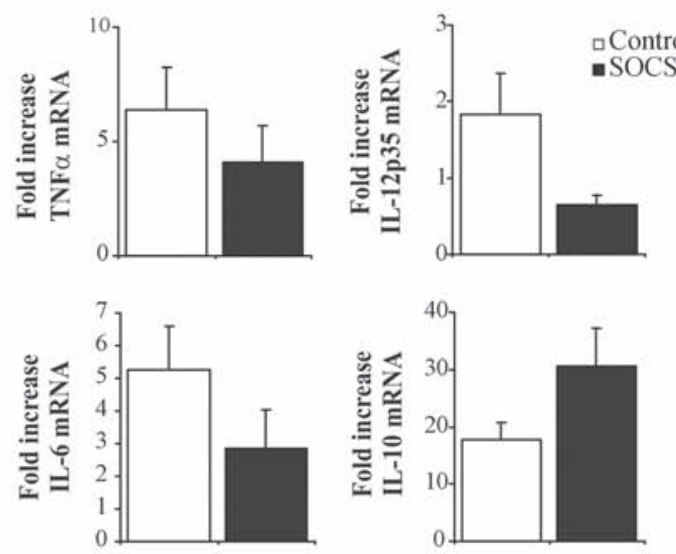

C
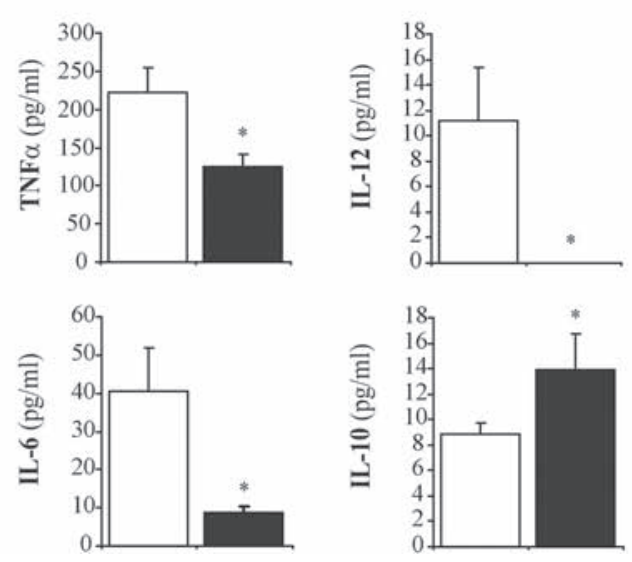

Figure 5 Altered cytokine profile of splenic antigen-presenting cells (APCs) after SOCS3 treatment. One day after the booster injection of type II collagen, DBA/1 mice were intravenously injected with $3 \times 10^{8}$ focusforming units (FFU) Ad5.SOCS3 or Ad5.luciferase. (A) Primary splenic APCs were obtained 1 or 3 days after virus injection and stained with CD1d monoclonal antibody. Filled histograms show isotype control. Data are shown as mean fluorescence intensity and representative results of two independent experiments ( $n=4$ mice per group). (B,C) Primary splenic APCs were obtained 1 day after virus injection and stimulated for $24 \mathrm{~h}$ with $1 \mu \mathrm{g} / \mathrm{ml}$ lipopolysaccharide (LPS). (B) Cells were harvested and mRNA expression levels of tumour necrosis factor $\alpha$ (TNF $\alpha)$, interleukin (IL)-6, IL-12p35 and IL-10 were determined by quantitative PCR. (C) Cytokine production was determined in cell-free supernatants of these cultures by Luminex multi-analyte technology. Values are mean and SEM results from triplicate cultures ( $n=4$ mice). ${ }^{*} p<0.05$ vs control. this inhibition was an altered cytokine release; decreased production of proinflammatory cytokines and enhanced production of anti-inflammatory IL-10 was found. Our findings clearly show that SOCS3 represses rather than deviates iNKT cell responses by inhibiting the immunostimulatory capacities of APCs.

Tissue-specific microenvironments like the local cytokine milieu are known to influence the behaviour of iNKT cells, which mainly depends on TLR engagement on the APC. ${ }^{26} 30$ Interestingly, SOCS3-transduced APCs showed a relatively weak capacity to initiate TLR-induced IL-2 production by the iNKT cell hybridoma. The activation of the iNKT cell hybridoma evoked by TLR4 interaction with APCs appeared to be independent of a direct invariant TCR-CD1d interaction. It is well known that IL-12 and IL-18 can enhance IFN $\gamma /$ IL-4 production by iNKT cells. ${ }^{31-33}$ Moreover, IL-10 is a powerful inhibitor of antigen-specific $\mathrm{T}$ cell proliferation and protects the host from exacerbated $\mathrm{T}$ cell responses during inflammation. ${ }^{34-36} \mathrm{~A}$ dominant role for endogenous IL-10 in the natural suppression of CIA has been demonstrated using neutralising antibodies. ${ }^{37}$ It is therefore likely that the diminished IL-12 and IL-18 levels together with the enhanced IL-10 levels play an important role in the SOCS3-mediated inhibitory effect on iNKT cell activity. Given the fact that IL-10 is capable of inducing SOCS3 expression, ${ }^{38} 39$ it is interesting to note that enhanced expression of SOCS3 by the iNKT cells results in reduced cytokine production. ${ }^{40}$

In addition to the antigen-independent activation, SOCS3transduced APCs were also less efficient in priming the iNKT cell hybridoma in an antigen-dependent manner. Although enhanced cell surface CD1d levels increase the efficiency of iNKT cell activation, ${ }^{29}$ we observed no differences in CD1d expression on splenic APCs from both groups. Interestingly, ligand activation of iNKT cells by DCs results in upregulation of CD40 ligand expression on the iNKT cells, which subsequently can stimulate DC maturation and IL-12 production. ${ }^{41-43}$ Furthermore, a recent study by Uemura et al showed that ligandactivated iNKT cells modify the IL-12p70/IL-23 balance in DCs mainly via the production of both Th1 and Th2 cytokines. ${ }^{44}$ This suggests that suboptimal stimulatory signals and soluble factors from $\alpha$-GalCer-activated iNKT cells (eg, IL-2, IFN $\gamma$ and/ or IL-4) can have a reciprocal stimulatory effect on the APCs in our co-culture system, which in turn can be inhibited in the SOCS3-transduced APCs. The effect of SOCS3 on the antigendependent activation of iNKT cells is therefore probably not caused by diminished antigen presentation but by changes in the cytokine microenvironment to which the APCs are exposed.

As reported previously, ${ }^{17}$ we have confirmed that intravenous delivery of adenovirus-mediated SOCS3 clearly prevents the development of CIA. There is extensive evidence that iNKT cells may influence the development and progression of experimental arthritis, but this may be beneficial or harmful to the host. In the context of this heterogeneity, variables that should be taken into account are the arthritis model studied, mode of iNKT cell activation, the stage of the disease and the genetic backgrounds between lines of mice. ${ }^{8} 45$ After collagen immunisation, iNKT cells undergo activation leading to enhanced percentages, cytokine release and expression of the early activation marker CD69. ${ }^{11}{ }^{46}$ Evidence has been accumulated that CD69 promotes lymphocyte retention in lymphoid organs via inhibition of the sphingosine-1-phosphate (S1P)S1PR axis. ${ }^{47}$ The enhanced CD69 expression on the iNKT cells from control virus-treated mice may therefore delay exit from the lymphoid organs during the 1-7-day period after virus injection. We demonstrated that in vivo delivery of SOCS3 
A

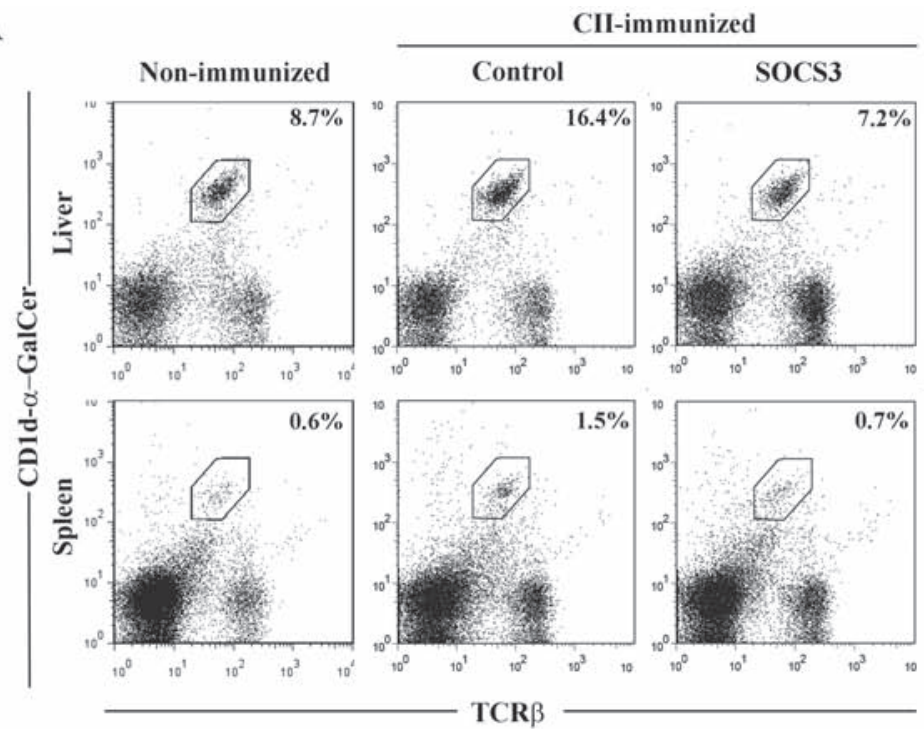

C

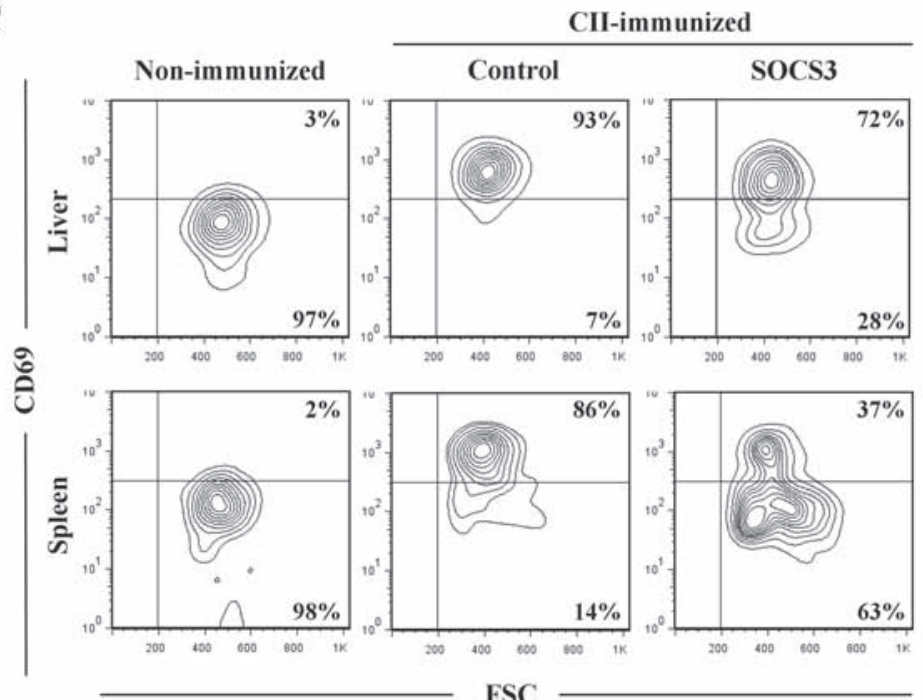

E

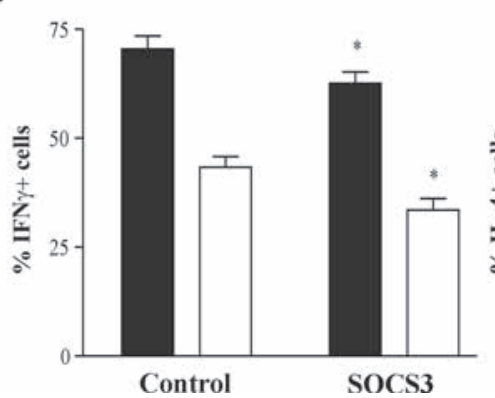

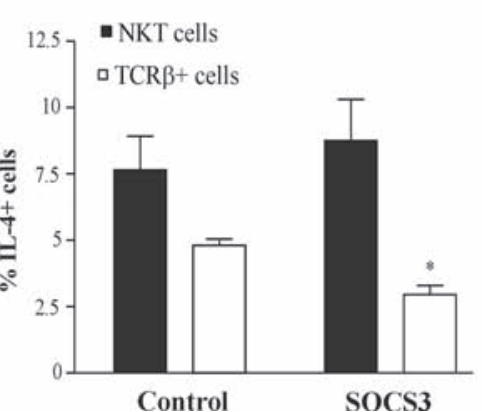

B
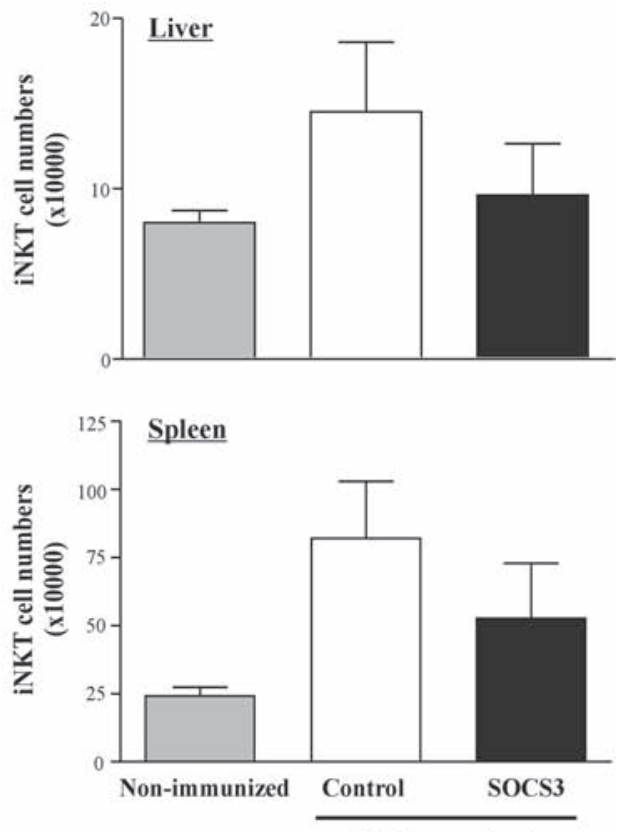

CII-immunized

D

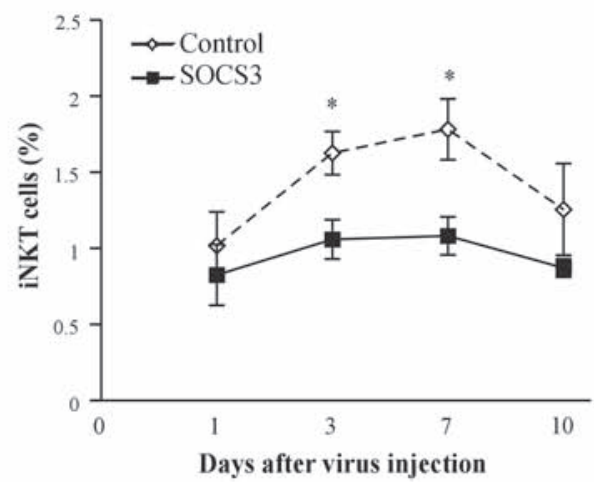

F

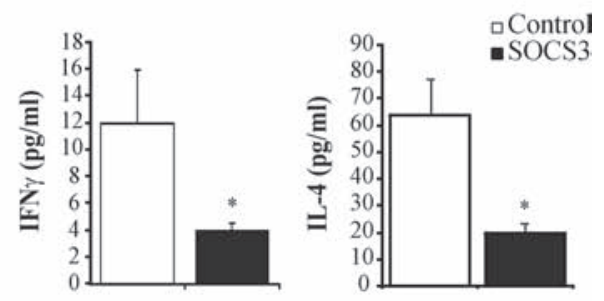

Figure 6 Intravenous delivery of adenovirus-mediated SOCS3 affects the in vivo function of invariant natural killer T (iNKT) cells during collagen-induced arthritis. Bovine type II collagen-immunised mice were intravenously injected with $3 \times 10^{8} \mathrm{FFU}$ of Ad5.SOCS3 or Ad5.luciferase and killed 3 days later. Non-immunised DBA/1 mice were used as a control. (A) Liver mononuclear cells and splenocytes were stained with T cell receptor $\beta$ (TCR $\beta$ ) monoclonal antibody and an $\alpha$-galactosylceramide ( $\alpha$-GalCer)-CD1d tetramer. Representative results of two independent experiments are shown. (B) Absolute numbers of iNKT cells in liver and spleen were calculated from the total liver mononuclear cells or splenocytes, respectively. Data are presented as mean and SEM ( $n=5$ mice). (C) Gated TCR $\beta+\alpha$-GalCer-CD1d tetramer-positive cells were screened for the expression of CD69. The data shown are of one representative experiment out of two with comparable results. (D) The iNKT cell frequency was determined in spleen during the 1-10-day period after virus injection. Data are presented as mean and SEM ( $\mathrm{n}=5$ mice). ${ }^{*} p<0.05$ vs control. (E) Hepatic TCR $\beta+$ cells and iNKT cells were stained for intracellular interferon $\gamma$ (IFN $\gamma$ ) and interleukin (IL)-4 production 3 days after virus injection and the percentage of positive cells are shown. Data are presented as mean and SEM ( $n=4$ mice). ${ }^{*} p<0.05$ vs control. (F) Liver mononuclear cells were stimulated for $48 \mathrm{~h}$ with $100 \mathrm{ng} / \mathrm{ml} \alpha$-GalCer. The levels of IFN $\gamma$ and IL-4 in cell-free supernatants were determined by Luminex multi-analyte technology. Data are presented in $\mathrm{pg} / \mathrm{ml}$ as mean and SEM of triplicate cultures $(\mathrm{n}=4$ mice). ${ }^{*} \mathrm{p}<0.05$ vs control. 
prevented the expansion of splenic and hepatic iNKT cells, inhibited the upregulation of CD69 expression and caused reduced production of IFN $\gamma$ and IL- 4 by LMNCs following $\alpha$-GalCer stimulation. These results show that SOCS3 does not cause a shift in iNKT phenotype but rather represses iNKT cell responses during the initiation phase of CIA. Together with the prophylactic effects of SOCS3, these findings suggest that iNKT cells have a stimulatory role in our study at this stage of the disease.

Besides iNKT cells, SOCS3-transduced APCs are also capable of inhibiting the type II collagen-specific T cell proliferation and IL-4, IFN $\gamma$ and IL-17 production by splenic CD3+ T cells. ${ }^{17}$ It is known that activation of iNKT cells can lead to downstream activation of other cell types in the immune system, including $T$ cells. Several studies have shown that in vivo administration of $\alpha$-GalCer with or without various protein antigens can lead to enhanced bystander activation of both CD4+ and/or CD8+ T cells. ${ }^{48} 49$ Whether SOCS3 overexpression also affects the bystander activation of $\mathrm{T}$ cells as another mechanism to regulate the immune response during CIA is still unknown and awaits further studies. Nevertheless, it is conceivable that the iNKT cells determine the threshold of immune activation and eventually the outcome of CIA induction.

In conclusion, this study clearly shows that SOCS3 inhibits the immunostimulatory function of APCs resulting in repression of iNKT cell responses during the initiation of CIA. Not only do these data substantiate the immunomodulatory capacities of SOCS3, they also give new insights into the possible endogenous role of iNKT cells in arthritis. The results of this study demonstrate a critical role for APCs in regulating iNKT cell function and suggest that genetic modification of APCs may be exploited as a therapeutic approach to modulate iNKT cell immune responses in vivo. The challenge will be to determine whether this strategy combined with $\alpha$-GalCer, or related analogues, results in superior therapeutic effects in autoimmune diseases such as rheumatoid arthritis.

Acknowledgements The authors thank the NHH Tetramer Core Facility (Atlanta, Georgia, USA) for providing CD1d/PBS57 tetramer, Dr E Nieuwenhuis (Erasmus Medical Centre, Sophia Children's Hospital, The Netherlands) for the gift of the iNKT cell hybridoma DN32.D3, Dr S Kochanek (Center for Molecular Medicine, University of Cologne, Germany) for kindly providing the N52E6 viral packaging cell line and Dr A van Spriel for critical reading of the manuscript.

Funding This study was financially supported by the Dutch Arthritis Association (Grant Number 01-1-304) and Radboud University Nijmegen Medical Centre. FAJvdL is supported by the Dutch Organization for Scientific Research (Grant Number 917.46.363).

\section{Competing interest None.}

Provenance and peer review Not commissioned; externally peer reviewed

\section{REFERENCES}

1. Godfrey DI, MacDonald HR, Kronenberg M, et al. NKT cells: what's in a name? Nat Rev Immunol 2004:4:231-7.

2. Matsuda JL, Naidenko OV, Gapin L, et al. Tracking the response of natural killer T cells to a glycolipid antigen using CD1d tetramers. J Exp Med 2000;192:741-54.

3. Bendelac A. CD1: presenting unusual antigens to unusual T lymphocytes. Science 1995;269:185-6

4. Lantz $\mathbf{0}$, Bendelac A. An invariant T cell receptor alpha chain is used by a unique subset of major histocompatibility complex class I-specific CD4+ and CD4-8- T cells in mice and humans. J Exp Med 1994;180:1097-106.

5. Kawano T, Cui J, Koezuka Y, et al. CD1d-restricted and TCR-mediated activation of valpha14 NKT cells by glycosylceramides. Science 1997;278:1626-9.

6. Park SH, Benlagha K, Lee D, et al. Unaltered phenotype, tissue distribution and function of Valpha14(+) NKT cells in germ-free mice. Eur J Immunol 2000;30:620-5.

7. Matsuda JL, Mallevaey T, Scott-Browne J, et al. CD1d-restricted iNKT cells, the 'Swiss-Army knife' of the immune system. Curr Opin Immunol 2008:20:358-68.

8. Drennan MB, Aspeslagh S, Elewaut D. Invariant natural killer T cells in rheumatic disease: a joint dilemma. Nat Rev Rheumato/ 2010;6:90-8.
9. Coppieters K, Van Beneden K, Jacques P, et al. A single early activation of invariant NK T cells confers long-term protection against collagen-induced arthritis in a ligandspecific manner. J Immunol 2007;179:2300-9.

10. Miellot A, Zhu R, Diem S, et al. Activation of invariant NK T cells protects against experimental rheumatoid arthritis by an IL-10-dependent pathway. Eur J Immunol 2005;35:3704-13.

11. Chiba A, Kaieda S, Oki S, et al. The involvement of V(alpha) 14 natural killer T cells in the pathogenesis of arthritis in murine models. Arthritis Rheum 2005;52:1941-8.

12. Kim HY, Kim HJ, Min HS, et al. NKT cells promote antibody-induced joint inflammation by suppressing transforming growth factor beta1 production. J Exp Med 2005;201:41-7

13. Trappeniers $\mathbf{M}$, Goormans $\mathbf{S}$, Van Beneden $\mathrm{K}$, et al. Synthesis and in vitro evaluation of alpha-GalCer epimers. ChemMedChem 2008;3:1061-70.

14. Ilangumaran S, Ramanathan S, Rottapel R. Regulation of the immune system by SOCS family adaptor proteins. Semin Immunol 2004;16:351-65.

15. Mansell A, Smith R, Doyle SL, et al. Suppressor of cytokine signaling 1 negatively regulates Toll-like receptor signaling by mediating Mal degradation. Nat Immunol 2006; 7:148-55.

16. Yoshimura A, Naka T, Kubo M. SOCS proteins, cytokine signalling and immune regulation. Nat Rev Immunol 2007;7:454-65.

17. Veenbergen S, Bennink MB, de Hooge AS, et al. Splenic suppressor of cytokine signaling 3 transgene expression affects $T$ cell responses and prevents development of collagen-induced arthritis. Arthritis Rheum 2008;58:3742-52.

18. Bendelac A, Lantz 0, Quimby ME, et al. CD1 recognition by mouse NK1+ T lymphocytes. Science 1995;268:863-5.

19. Geurts J, Joosten LA, Takahashi N, et al. Computational design and application of endogenous promoters for transcriptionally targeted gene therapy for rheumatoid arthritis. Mol Ther 2009;17:1877-87.

20. Chartier C, Degryse E, Gantzer M, et al. Efficient generation of recombinant adenovirus vectors by homologous recombination in Escherichia coli. J Virol 1996;70:4805-10.

21. Smeets RL, van de Loo FA, Joosten $L A$, et al. Effectiveness of the soluble form of the interleukin-1 receptor accessory protein as an inhibitor of interleukin-1 in collagen-induced arthritis. Arthritis Rheum 2003:48:2949-58.

22. Chomczynski P, Sacchi N. Single-step method of RNA isolation by acid guanidinium thiocyanate-phenol-chloroform extraction. Anal Biochem 1987;162:156-9

23. Smeets RL, Veenbergen S, Arntz OJ, et al. A novel role for suppressor of cytokine signaling 3 in cartilage destruction via induction of chondrocyte desensitization toward insulin-like growth factor. Arthritis Rheum 2006;54:1518-28.

24. Brigl M, Bry L, Kent SC, et al. Mechanism of CD1d-restricted natural killer T cell activation during microbial infection. Nat Immunol 2003;4:1230-7.

25. Sada-Ovalle I, Chiba A, Gonzales A, et al. Innate invariant NKT cells recognize Mycobacterium tuberculosis-infected macrophages, produce interferon-gamma, and kill intracellular bacteria. PLoS Pathog 2008; 4:e1000239.

26. Nagarajan NA, Kronenberg M. Invariant NKT cells amplify the innate immune response to lipopolysaccharide. J Immunol 2007;178:2706-13.

27. Johnson M, Huyn S, Burton J, et al. Differential biodistribution of adenoviral vector in vivo as monitored by bioluminescence imaging and quantitative polymerase chain reaction. Hum Gene Ther 2006;17:1262-9.

28. Stone D, Liu Y, Shayakhmetov D, et al. Adenovirus-platelet interaction in blood causes virus sequestration to the reticuloendothelial system of the liver. J Virol 2007:81:4866-71.

29. Sköld M, Xiong X, Illarionov PA, et al. Interplay of cytokines and microbial signals in regulation of CD1d expression and NKT cell activation. J Immunol 2005:175:3584-93.

30. Mattner J, Debord KL, Ismail N, et al. Exogenous and endogenous glycolipid antigens activate NKT cells during microbial infections. Nature 2005:434:525-9

31. Baxevanis CN, Gritzapis AD, Papamichail M. In vivo antitumor activity of NKT cells activated by the combination of IL-12 and IL-18. J Immunol 2003;171:2953-9.

32. Zhu R, Diem S, Araujo LM, et al. The Pro-Th1 cytokine IL-12 enhances IL-4 production by invariant NKT cells: relevance for T cell-mediated hepatitis. J Immunol 2007; 178:5435-42

33. Leite-De-Moraes MC, Hameg A, Pacilio M, et al. IL-18 enhances IL-4 production by ligand-activated NKT Iymphocytes: a pro-Th2 effect of IL-18 exerted through NKT cells. J Immunol 2001;166:945-51.

34. de Waal Malefyt R, Haanen J, Spits H, et al. Interleukin 10 (IL-10) and viral IL-10 strongly reduce antigen-specific human $\mathrm{T}$ cell proliferation by diminishing the antigen-presenting capacity of monocytes via downregulation of class II major histocompatibility complex expression. J Exp Med 1991;174:915-24.

35. Li MO, Flavell RA. Contextual regulation of inflammation: a duet by transforming growth factor-beta and interleukin-10. Immunity 2008;28:468-76.

36. Taga K, Tosato G. IL-10 inhibits human T cell proliferation and IL-2 production. J Immunol 1992:148:1143-8. 
37. Joosten LA, Lubberts E, Durez P, et al. Role of interleukin-4 and interleukin-10 in murine collagen-induced arthritis. Protective effect of interleukin-4 and interleukin-10 treatment on cartilage destruction. Arthritis Rheum 1997;40:249-60.

38. Cassatella MA, Gasperini S, Bovolenta C, et al. Interleukin-10 (IL-10) selectively enhances CIS3/SOCS3 mRNA expression in human neutrophils: evidence for an IL-10-induced pathway that is independent of STAT protein activation. Blood 1999:94:2880-9.

39. Niemand C, Nimmesgern A, Haan S, et al. Activation of STAT3 by IL-6 and IL-10 in primary human macrophages is differentially modulated by suppressor of cytokine signaling 3. J Immunol 2003;170:3263-72.

40. Nakaya M, Hashimoto M, Nakagawa R, et al. SOCS3 in T and NKT cells negatively regulates cytokine production and ameliorates ConA-induced hepatitis. J Immunol 2009; 183:7047-53.

41. Tomura M, Yu WG, Ahn HJ, et al. A novel function of Valpha14+CD4+NKT cells: stimulation of IL-12 production by antigen-presenting cells in the innate immune system. J Immunol 1999:163:93-101.

42. Kitamura $\mathbf{H}$, Iwakabe K, Yahata T, et al. The natural killer T (NKT) cell ligand alpha-galactosylceramide demonstrates its immunopotentiating effect by inducing interleukin (IL)-12 production by dendritic cells and IL-12 receptor expression on NKT cells. J Exp Med 1999;189:1121-8.
43. Vincent MS, Leslie DS, Gumperz JE, et al. CD1-dependent dendritic cell instruction. Nat Immunol 2002;3:1163-8.

44. Uemura Y, Liu TY, Narita Y, et al. Cytokine-dependent modification of IL-12p70 and IL-23 balance in dendritic cells by ligand activation of Valpha24 invariant NKT cells. $\mathrm{J}$ Immunol 2009:183:201-8.

45. Teige A, Bockermann R, Hasan M, et al. CD1d-dependent NKT cells play a protective role in acute and chronic arthritis models by ameliorating antigen-specific Th1 responses. J Immunol 2010;185:345-56.

46. Miellot-Gafsou A, Biton J, Bourgeois E, et al. Early activation of invariant natural killer T cells in a rheumatoid arthritis model and application to disease treatment. Immunology 2010;130:296-306.

47. Spiegel S, Milstien S. The outs and the ins of sphingosine-1-phosphate in immunity. Nat Rev Immunol 2011;11:403-15.

48. Nishimura T, Kitamura $\mathrm{H}$, Iwakabe $\mathrm{K}$, et al. The interface between innate and acquired immunity: glycolipid antigen presentation by CD1d-expressing dendritic cells to NKT cells induces the differentiation of antigen-specific cytotoxic T lymphocytes. Int Immunol 2000;12:987-94.

49. Singh N, Hong S, Scherer DC, et al. Cutting edge: activation of NK T cells by CD1d and alpha-galactosylceramide directs conventional T cells to the acquisition of a Th2 phenotype. J Immuno/ 1999;163:2373-7. 